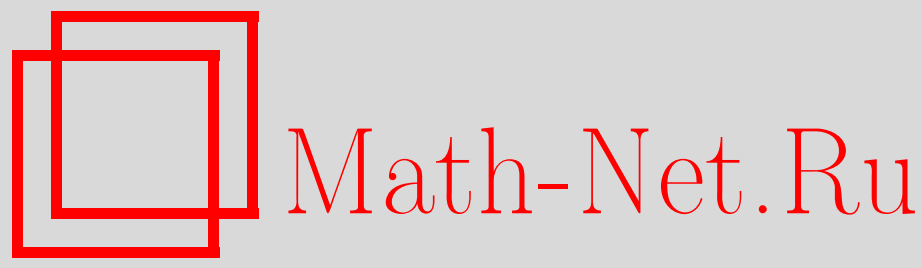

В. Г. Марихин, В. В. Соколов, О некоторых интегральных уравнениях, связанных со случайными гауссовскими процессами, ТМФ, 2010, том 164, номер 2, 196-206

DOI: https://doi.org/10.4213/tmf6533

Использование Общероссийского математического портала Math-Net.Ru подразумевает, что вы прочитали и согласны с пользовательским соглашением http://www.mathnet.ru/rus/agreement

Параметры загрузки:

IP: 54.198 .67 .100

26 апреля 2023 г., 13:58:56

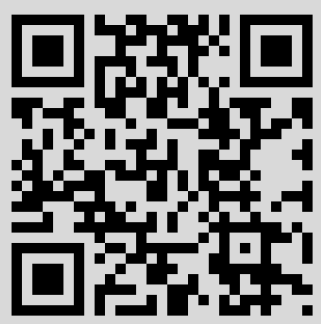




\title{
ФИЗИКА
}

Том 164, № 2

август, 2010

\section{О НЕКОТОРЫХ ИНТЕГРАЛЬНЫХ УРАВНЕНИЯХ, СВЯЗАННЫХ СО СЛУЧАЙНЫМИ ГАУССОВСКИМИ ПРОЦЕССАМИ}

\begin{abstract}
Для вычисления преобразования Лапласа от интеграла квадрата случайного гауссовского процесса рассматривается нелинейное интегральное уравнение типа уравнения Вольтерра. Это уравнение является тождеством Уорда для производящей корреляционной функции. Оказывается, что для некоторого важного класса корреляционных функций оно сводится к линейному обыкновенному дифференциальному уравнению. Приводятся достаточные условия интегрируемости этого уравнения (коэффициенты уравнения постоянны). Для ряда конкретных случайных гауссовских процессов таких, как модель "броуновского моста" и модель Орнштейна-Уленбека, преобразование Лапласа получено точно.
\end{abstract}

Ключевые слова: случайные процессы, интегральные уравнения, преобразование Лапласа.

\section{1. ВВЕДЕНИЕ}

При изучении случайных гауссовских процессов возникает задача о вычислении высших корреляционных функций. Поскольку процесс является гауссовским, нет никаких проблем при вычислении любой корреляционной функции заданного порядка. Действительно, среднее (или математическое ожидание) от произведения нечетного числа случайных полей равно нулю, а среднее от четного числа полей вычисляется по правилу Вика (т.е. равно сумме всех попарных средних):

$$
\begin{aligned}
E(x(t))=0, \quad & E\left(x\left(t_{1}\right) x\left(t_{2}\right) \ldots x\left(t_{2 n+1}\right)\right)=0, \\
E\left(x\left(t_{1}\right) x\left(t_{2}\right) \ldots x\left(t_{2 n}\right)\right)= & E\left(x\left(t_{1}\right) x\left(t_{2}\right)\right) E\left(x\left(t_{3}\right) x\left(t_{4}\right)\right) \cdots+ \\
& +E\left(x\left(t_{1}\right) x\left(t_{3}\right)\right) E\left(x\left(t_{2}\right) x\left(t_{4}\right)\right) \cdots+\cdots .
\end{aligned}
$$

Здесь и далее $E(a)$ - среднее от $a$ по случайному процессу. Однако для ряда приложений бывает важно иметь информацию о виде корреляций сразу во всех порядках

${ }^{*}$ Институт теоретический физики им. Л. Д. Ландау РАН, Москва, Россия. E-mail: mvg@itp.ac.ru, sokolov@itp.ac.ru 
по числу входящих в корреляционную функцию случайных полей, для чего можно ввести производящую функцию, например преобразование Лапласа интеграла квадрата случайного процесса $x(t)$ :

$$
L=E e^{-\lambda \int_{0}^{t} x^{2}(s) d s} .
$$

Действительно, при разложении в ряд Тейлора выражения (2) по степеням $\lambda$ можно получить $2 n$-точечную корреляционную функцию с попарно совпадающими аргументами.

Оказывается, что преобразование Лапласа (2) может быть найдено с помощью решения нелинейного интегрального уравнения типа уравнения Вольтерра. А именно, имеет место следующая теорема.

Теорема 1. Для любого гауссовского процесса $x(t)$

$$
L=e^{-\int_{0}^{t} \gamma(s, s) d s / 2},
$$

где $\gamma(t, s)$ удовлетворяет интегральному уравнению

$$
\gamma(t, s)=2 \lambda K(t, s)-\int_{0}^{s} \gamma(t, k) \gamma(s, k) d k, \quad s \leqslant t .
$$

Здесь $K(t, s)=E(x(t) x(s))$ - корреляционная функция процесса $x(t)$. Отметим, что порядок аргументов у подынтегральных функций нетипичен для нелинейных уравнений типа Вольтерра.

Теорема 1 была доказана впервые в работе [1] методами теории вероятности. В конце нашей работы мы приведем другое доказательство, использующее определение случайного гауссовского процесса с помощью часто используемого в физических приложениях метода функционального интеграла.

Кроме того, оказывается [2], что функция $\gamma(s, s)$ является ошибкой фильтрации в линейной схеме

$$
y(t)=\int_{0}^{t} x(s) d s+w(t)
$$

где $x(t)$ - гауссовский сигнал с корреляционной функцией $K(t, s), w(t)$ - винеровский шум. Сама функция $\gamma(t, s)$ фигурирует в формуле для фильтра.

Для нескольких простейших моделей функция $K(t, s)$ при $s \leqslant t$ имеет вид

$$
K(t, s)=\sum_{i=1}^{n} \Psi_{i}(t) \Phi_{i}(s)
$$

Основным достоинством интегрального уравнения (3) является то, что функция $K(t, s)$ фигурирует в нем только при $s \leqslant t$, следовательно, для работы с этим уравнением нам достаточно формулы (4).

Корреляционные функции имеют вид (4), например, для следующих моделей [3], [4]:

- винеровского процесса $K(t, s)=s$;

- модели "броуновского моста" $K(t, s)=s(1-t)$;

- (стационарной) модели Орнштейна-Уленбека $K(t, s)=e^{s-t}$. 
Кроме того, если корреляционная функция процесса $x(t)$ имеет вид (4), то корреляционная функция $\widehat{K}$ от интеграла $y(t)=\int_{0}^{t} x(s) d s$ имеет ту же структуру и находится по формуле

$$
\widehat{K}(t, s)=\sum_{i=1}^{n+1} \widehat{\Psi}_{i}(t) \widehat{\Phi}_{i}(s), \quad s \leqslant t
$$

где

$$
\widehat{\Psi}_{i}(t)=\int_{0}^{t} \Psi_{i}(k) d k, \quad \widehat{\Phi}_{i}(s)=\int_{0}^{s} \Phi_{i}(k) d k
$$

при $i=1,2, \ldots, n$ и

$$
\widehat{\Psi}_{n+1}(t)=1, \quad \widehat{\Phi}_{n+1}(s)=\int_{0}^{s}\left(\Psi^{\mathrm{T}}(k) \int_{0}^{k} \Phi(r) d r-\Phi^{\mathrm{T}}(k) \int_{0}^{k} \Psi(r) d r\right) d k
$$

Здесь $\Psi$ и $\Phi$ - векторы-столбцы, составленные из функций $\Psi_{i}$ и $\Phi_{i}$, Т означает обычное транспонирование матриц. $\mathrm{K}$ примеру, корреляционная функция интеграла для винеровского процесса при $s \leqslant t$ имеет вид $K(t, s)=t s^{2} / 2-s^{3} / 6$.

Во всех приведенных выше примерах функция (4) обладает следующим замечательным свойством:

$$
K(t, s)=K_{1}(t, s)+K_{2}(t-s), \quad K_{1}(t, s)=K_{1}(s, t), \quad K_{2}(-z)=-K_{2}(z) .
$$

Другими словами, при разложении корреляционной функции при $s \leqslant t$ в сумму симметрической и кососимметрической функций последняя зависит только от разности $t-s$.

Кроме того, для рассмотренных примеров $K(t, s)$ является квазиполиномом от двух переменных, т.е. имеет вид

$$
K(t, s)=\sum_{i} P_{i}(t, s) e^{\lambda_{i} t+\mu_{i} s}, \quad s \leqslant t
$$

где $P_{i}(t, s)$ - некоторые многочлены над $\mathbb{C}, \lambda_{i}, \mu_{i}$ - (вообще говоря, комплексные) постоянные. Очевидно, что каждый квазиполином имеет вид (4).

В настоящей работе мы показываем, что для процесса с корреляционной функцией вида (7), удовлетворяющей условию (6), уравнение (3) интегрируется в элементарных функциях. При этом функция $\gamma(s, s)$ имеет вид

$$
\gamma(s, s)=\frac{d}{d s} \ln F(s)
$$

где $F$ - некоторый квазиполином.

В качестве примеров функция $F(s)$ найдена явно для винеровского процесса (формула Камерона-Мартина [5]), модели "броуновского моста", их интегралов и стационарной модели Орнштейна-Уленбека (см. также работу [6]). 


\section{2. МАТРИЧНОЕ УРАВНЕНИЕ РИККАТИ}

Рассмотрим интегральное уравнение (3), где $K(t, s)$ имеет вид (4). В этом и следующем разделах для краткости мы полагаем $\lambda=1 / 2$. В примерах из раздела 3 зависимость от $\lambda$ будет восстановлена и множитель $2 \lambda$ включен в вектор $\Phi$.

Будем искать решение в виде

$$
\gamma(t, s)=\sum_{i=1}^{n} \Psi_{i}(t) f_{i}(s) .
$$

Легко видеть, что функции $f_{i}(s)$ находятся из системы интегральных уравнений

$$
f_{i}(s)=\Phi_{i}(s)-\sum_{j=1}^{n} \Psi_{j}(s) J_{i j},
$$

где

$$
J_{i j}=\int_{0}^{s} f_{i}(k) f_{j}(k) d k .
$$

Рассмотрим интегралы $J_{i j}$ как новые неизвестные. Тогда

$$
J_{i j}^{\prime}=f_{i} f_{j}=\left(\Phi_{i}(s)-\sum_{k=1}^{n} \Psi_{k}(s) J_{i k}\right)\left(\Phi_{j}(s)-\sum_{k=1}^{n} \Psi_{k}(s) J_{j k}\right) .
$$

Последнюю систему можно записать в компактном матрично-векторном виде:

$$
J^{\prime}=(J \Psi-\Phi)\left(\Psi^{\mathrm{T}} J-\Phi^{\mathrm{T}}\right), \quad J(0)=0,
$$

где $J$ - неизвестная симметрическая матрица с элементами $J_{i j}$. Нетрудно проверить, что если $K(t, s)$ имеет вид (4), то уравнение (3) эквивалентно задаче Коши (11). Отметим, что при вычислении преобразования Лапласа от интеграла квадрата гауссовского процесса нас интересует не матрица $J(s)$, а скаляр

$$
\gamma(s, s)=\Psi^{\mathrm{T}} \Phi-\Psi^{\mathrm{T}} J \Psi .
$$

Система (11), как и любое матричное уравнение Риккати

$$
J^{\prime}=J A(s) J+B(s) J+J C(s)+D(s),
$$

где $A, B, C, D$ - матрицы размера $n \times n$, может быть сведено к системе линейных уравнений с переменными коэффициентами.

А именно, нетрудно проверить, что если матрицы $y_{1}$ и $y_{2}$ размера $n \times n$ удовлетворяют линейной системе

$$
\left(\begin{array}{l}
y_{1} \\
y_{2}
\end{array}\right)^{\prime}=\left(\begin{array}{cc}
B & D \\
-A & -C
\end{array}\right)\left(\begin{array}{l}
y_{1} \\
y_{2}
\end{array}\right)
$$

то $J=y_{1} y_{2}^{-1}$ удовлетворяет уравнению (13). 
Линейная система, соответствующая уравнению (11), имеет вид

$$
\left(\begin{array}{l}
y_{1} \\
y_{2}
\end{array}\right)^{\prime}=\left(\begin{array}{ll}
-\Phi \Psi^{\mathrm{T}} & \Phi \Phi^{\mathrm{T}} \\
-\Psi \Psi^{\mathrm{T}} & \Psi \Phi^{\mathrm{T}}
\end{array}\right)\left(\begin{array}{l}
y_{1} \\
y_{2}
\end{array}\right) .
$$

Поскольку у нас имеется дополнительное условие $J(0)=0$, то нужно найти такое решение системы $(15)$, что $y_{1}(0)=0$.

Специфика системы (15) состоит в том, что

$$
y_{1}^{\prime}=\Phi h^{\mathrm{T}}, \quad y_{2}^{\prime}=\Psi h^{\mathrm{T}},
$$

где вектор $h(s)$ задается формулой

$$
h=-y_{1}^{\mathrm{T}} \Psi+y_{2}^{\mathrm{T}} \Phi .
$$

ПРЕДЛОЖЕНИЕ. Имеет место формула

$$
\gamma(s, s)=\frac{d}{d s} \ln \operatorname{det} y_{2}
$$

ДокАЗАтельство. Умножим равенство $h^{\mathrm{T}}=-\Psi^{\mathrm{T}} y_{1}+\Phi^{\mathrm{T}} y_{2}$ справа на $y_{2}^{-1}$. Получим $h^{\mathrm{T}} y_{2}^{-1}=-\Psi^{\mathrm{T}} J+\Phi^{\mathrm{T}}$. Умножив это равенство справа на $\Psi$, находим (см. (12)), что

$$
\gamma(s, s)=h^{\mathrm{T}} y_{2}^{-1} \Psi .
$$

Умножим последнее равенство справа на $h^{\mathrm{T}}$ и воспользуемся второй из формул (16). В результате получаем, что $\Gamma(s)$ является (ненулевым) собственным значением матрицы $Q=\left(y_{2}^{-1} y_{2}^{\prime}\right)^{\mathrm{T}}$. В силу уравнений (16) матрица $y_{2}^{\prime}$ имеет ранг 1. Поэтому ранг матрицы $Q$ также равен 1. Для такой матрицы (единственное) ненулевое собственное значение равно следу, т.е. $\gamma(s, s)=\operatorname{Sp} Q$. Теперь (18) следует из формулы Лиувилля для вронскиана.

\section{3. УСЛОВИЯ ТОЧНОЙ ИНТЕГРИРУЕМОСТИ ДЛЯ КВАЗИПОЛИНОМИАЛЬНОЙ КОРРЕЛЯЦИОННОЙ ФУНКЦИИ}

Если нам известен вектор $h(s)$, то матрицы $y_{1}$ и $y_{2}$ находятся из уравнений $(16)$ интегрированием. Поэтому линейное дифференциальное уравнение, которому удовлетворяет $h(s)$, является ключевым во всех вопросах, связанных с уравнениями (11) и (15). Ниже предлагается относительно простой способ явного нахождения этого уравнения. Кроме того, мы приводим достаточное условие того, что уравнение для $h$ имеет постоянные коэффициенты.

Для всякой функции $K(t, s)$ обозначим

$$
N_{i}(s)=\left(\frac{\partial^{i}}{\partial t^{i}} K(t, s)-\frac{\partial^{i}}{\partial s^{i}} K(t, s)\right)(s, s) .
$$

В случае, когда $K(t, s)$ имеет вид (4),

$$
N_{i}=\Phi^{\mathrm{T}} \frac{d^{i}}{d s^{i}} \Psi-\Psi^{\mathrm{T}} \frac{d^{i}}{d s^{i}} \Phi .
$$


Ясно, что если выполнено условие (6), то

$$
N_{2 k-1}(s)=\alpha_{k}, \quad k=1,2, \ldots,
$$

где $\alpha_{k}$ - некоторые постоянные.

Теорема 2. Пусть $\Phi(s)$ u $\Psi(s)$ удовлетворяют некоторому линейнъму однородному дифференциальному уравнению с постоянными коэффициентами

$$
L(Z)=\sum_{i=0}^{m} \lambda_{i} \frac{d^{i}}{d s^{i}} Z=0 .
$$

Тогда, если выполнены условия (20), то вектор $h(s)$, заданный формулой (17), удовлетворяет уравнению

$$
\sum_{i=0}^{m} \lambda_{i} P_{i} h=0,
$$

где дифференциалъные операторы $P_{k}$ с постоянными коэффициентами определяются рекуррентными формулами

$$
P_{0}=1, \quad P_{2 i+1}=P_{2 i} \frac{d}{d s}, \quad P_{2 i+2}=P_{2 i} \frac{d^{2}}{d s^{2}}+\alpha_{i} .
$$

ДокАзАтЕльство. Будем дифференцировать соотношение (17), пользуясь тождествами (16) и (20). Получим

$$
\begin{aligned}
h^{\prime} & =-y_{1}^{\mathrm{T}} \Psi^{\prime}+y_{2}^{\mathrm{T}} \Phi^{\prime}, \\
h^{\prime \prime}+\alpha_{1} h & =-y_{1}^{\mathrm{T}} \Psi^{\prime \prime}+y_{2}^{\mathrm{T}} \Phi^{\prime \prime}, \\
\left(h^{\prime \prime}+\alpha_{1} h\right)^{\prime} & =-y_{1}^{\mathrm{T}} \Psi^{\prime \prime \prime}+y_{2}^{\mathrm{T}} \Phi^{\prime \prime \prime}, \\
\left(h^{\prime \prime}+\alpha_{1} h\right)^{\prime \prime}+\alpha_{2} h & =-y_{1}^{\mathrm{T}} \Psi^{\prime \prime \prime \prime}+y_{2}^{\mathrm{T}} \Phi^{\prime \prime \prime \prime}
\end{aligned}
$$

и т.д. Таким образом,

$$
-y_{1}^{\mathrm{T}} \Psi^{(k)}+y_{2}^{\mathrm{T}} \Phi^{(k)}=P_{k}(h) .
$$

Если $\Phi$ и $\Psi$ удовлетворяют уравнению (21), то из (23) следует (22).

ЗАмЕчание 1. Аналогично доказывается, что если условия (20) выполнены, а $\Phi$ и $\Psi$ удовлетворяют уравнению с переменными коэффициентами

$$
\sum_{i=0}^{m} r_{i}(s) \frac{d^{i}}{d s^{i}} Z=0,
$$

Tо

$$
\sum_{i=0}^{m} r_{i}(s) P_{i}(h)=0 .
$$

Условия (20) можно рассматривать как систему обыкновенных дифференциальных уравнений относительно неизвестных $\Psi_{i}, \Phi_{i}$. Решив ее, можно в принципе описать для заданного $n$ все функции (4), удовлетворяющие (20). 
Например, пусть $n=1$. Тогда $K(t, s)=\Psi(t) \Phi(s)$. С точки зрения теории вероятности это в точности означает, что мы рассматриваем гауссовские марковские процессы.

Если $\alpha_{1}=0$, то $\Psi(s)=$ const.$\Phi(s)$ и все условия (20) выполнены с $\alpha_{i}=0$. Соответствующий случайный процесс тривиален, т.е. имеет вид $x(t)=\Phi(t) \xi$, где $\xi-$ некоторая (постоянная) гауссовская случайная величина.

Если $\alpha_{1} \neq 0$, то рассмотрим линейное дифференциальное уравнение второго порядка, для которого $\Phi(s)$ и $\Psi(s)$ - пара линейно независимых решений. Поскольку вронскиан этих решений равен постоянной $\alpha_{1}$, уравнение имеет вид

$$
Z^{\prime \prime}=q(s) Z
$$

Условие $\Phi(s) \Psi(s)^{\prime \prime \prime}-\Psi(s) \Phi(s)^{\prime \prime \prime}=\alpha_{2}$ дает нам $q(s)=\alpha_{2} / \alpha_{1}$. При этом все условия (20) выполнены с $\alpha_{i}=\alpha_{2}^{i-1} \alpha_{1}^{2-i}, i=3,4, \ldots$.

Таким образом, если $\alpha_{2}=0$, то

$$
\Phi(s)=b_{1} s+b_{0}, \quad \Psi(s)=c_{1} s+c_{0} .
$$

Если же $\alpha_{2} \neq 0$, то

$$
\Phi(s)=b_{1} e^{k s}+b_{0} e^{-k s}, \quad \Psi(s)=c_{1} e^{k s}+c_{0} e^{-k s},
$$

где $k^{2}=\alpha_{2} / \alpha_{1}$. Гауссовские процессы с соответствующими корреляционными функциями часто встречаются в приложениях (см. раздел 1).

ОПРЕДЕЛЕНИЕ. Назовем случайный процесс с корреляционной функцией (4) интегрируемым, если для него выполнены условия теоремы 2.

Поскольку для интегрируемого процесса $h$ и $\Psi$ - квазиполиномы, то квазиполином является и $y_{2}=\int \Psi h^{\mathrm{T}} d s$. Поэтому согласно (18) искомая функция $\gamma(s, s)$ представляет собой логарифмическую производную от некоторого квазиполинома.

Теорема 3. Интеграл от любого интегрируемого процесса интегрируем.

ДокАЗАТЕЛЬСтво. Используя формулу (5), нетрудно проверить, что

$$
\widehat{N}_{1}=0, \quad \widehat{N}_{2 i-1}=-\alpha_{i-1}, \quad i=2,3, \ldots .
$$

ЗАмЕчание 2. Легко видеть, что бо́льшая часть результатов, изложеных выше, формально остается справедливой и для случая, когда сумма в формуле (4) бесконечна.

\section{4. ПРИМЕРЫ}

Рассмотрим случай, когда $n=1$, а $\Phi_{1}(s)$ и $\Psi_{1}(s)$ заданы формулами (24). При этом $\alpha_{1}=b_{0} c_{1}-b_{1} c_{0}$. Поскольку уравнение $(21)$ имеет вид $Z^{\prime \prime}=0$, имеем

$$
h^{\prime \prime}+\alpha_{1} h=0
$$


Для винеровского процесса $b_{1}=2 \lambda, b_{0}=0, c_{1}=0, c_{0}=1$. Следовательно, $\alpha_{1}=-2 \lambda$ и $h=\mu_{1} e^{k s}+\mu_{2} e^{-k s}$, где $k=\sqrt{2 \lambda}$. Формулы (16) дают

$$
y_{1}=\mu_{1}(k s-1) e^{k s}-\mu_{2}(k s+1) e^{-k s}, \quad y_{2}=\mu_{1} e^{k s}-\mu_{2} e^{-k s} .
$$

Условие $y_{1}(0)=0$ эквивалентно тому, что $\mu_{1}+\mu_{2}=0$. Формула (18) приводит к уравнению (8), где

$$
F(s)=\operatorname{ch}(\sqrt{2 \lambda} s) .
$$

Аналогичные вычисления для "броуновского моста" дают

$$
F(s)=\sqrt{2 \lambda} s \operatorname{ch}(\sqrt{2 \lambda} s)-e^{\sqrt{2 \lambda} s} .
$$

Для случая $n=2$

$$
\begin{array}{ll}
\Phi_{1}(s)=a_{3} s^{3}+a_{2} s^{2}+a_{1} s+a_{0}, & \Phi_{2}(s)=b_{3} s^{3}+b_{2} s^{2}+b_{1} s+b_{0}, \\
\Psi_{1}(s)=c_{3} s^{3}+c_{2} s^{2}+c_{1} s+c_{0}, & \Psi_{2}(s)=d_{3} s^{3}+d_{2} s^{2}+d_{1} s+d_{0} .
\end{array}
$$

Легко проверить, что если корреляционная функция (4) удовлетворяет условию (6), то уравнение $(22)$ для $h(s)$ имеет вид

$$
h^{\prime \prime \prime \prime}+\alpha_{1} h^{\prime \prime}+\alpha_{2} h=0,
$$

где $\alpha_{1}=a_{0} c_{1}-a_{1} c_{0}+b_{0} d_{1}-b_{1} d_{0}, \alpha_{2}=6\left(a_{0} c_{3}-a_{3} c_{0}+b_{0} d_{3}-b_{3} d_{0}\right)$.

В частности, для интеграла от винеровского процесса постоянные $a_{3}, a_{1}, a_{0}, b_{2}$, $b_{1}, b_{0}, c_{3}, c_{2}, c_{0}, d_{3}, d_{2}, d_{1}$ равны нулю и

$$
a_{2}=\lambda, \quad b_{3}=\frac{\lambda}{3}, \quad c_{1}=1, \quad d_{0}=-1 .
$$

Уравнение $(26)$ имеет вид $h^{\prime \prime \prime \prime}+2 \lambda h=0$ и $h=\left(h_{1}, h_{2}\right)^{\mathrm{T}}$, где

$$
\begin{aligned}
& h_{1}=\lambda_{1} e^{k s}+\lambda_{2} e^{i k s}+\lambda_{3} e^{-k s}+\lambda_{4} e^{-i k s}, \\
& h_{2}=\mu_{1} e^{k s}+\mu_{2} e^{i k s}+\mu_{3} e^{-k s}+\mu_{4} e^{-i k s},
\end{aligned}
$$

$k^{4}=-2 \lambda$. Условие $y_{1}(0)=0$ эквивалентно тому, что

$$
\begin{array}{ll}
\lambda_{1}+\lambda_{2}+\lambda_{3}+\lambda_{4}=0, & -\lambda_{1}-i \lambda_{2}+\lambda_{3}+i \lambda_{4}=0, \\
\mu_{1}+\mu_{2}+\mu_{3}+\mu_{4}=0, & -\mu_{1}-i \mu_{2}+\mu_{3}+i \mu_{4}=0 .
\end{array}
$$

Если константы $\lambda_{i}, \mu_{i}$ удовлетворяют этим соотношениям, то формула (18) дает

$$
F(s)=\operatorname{ch}(\mu s)+\cos (\mu s)+2, \quad \mu^{4}=8 \lambda .
$$

Для интеграла от “броуновского моста" вычисления приводят к следующему результату:

$$
F(s)=(\mu s-\sqrt{2})(\operatorname{ch}(\mu s)+\cos (\mu s)+2)-2(\operatorname{sh}(\mu s)+\sin (\mu s)) .
$$

В заключение найдем $F(s)$ для модели Орнштейна-Уленбека. В этом случае в формуле (25) нужно положить $k=1, b_{1}=2 \lambda, b_{0}=0, c_{1}=0, c_{0}=1$. Легко видеть, что все условия (20) выполнены, причем $N_{2 i-1}=-4 \lambda$ для любого $i$. Так как уравнение $(21)$ имеет вид $Z^{\prime \prime}=Z$, то $h^{\prime \prime}-(4 \lambda+1) h=0$. Вычисления, аналогичные предыдущим, дают

$$
F(s)=(k+1)^{2} e^{(k-1) s}-(k-1)^{2} e^{-(k+1) s}, \quad k=\sqrt{4 \lambda+1} .
$$




\section{5. ВЫЧИСЛЕНИЕ ПРЕОБРАЗОВАНИЯ ЛАПЛАСА МЕТОДОМ ФУНКЦИОНАЛЬНОГО ИНТЕГРАЛА}

Определение случайного гауссовского процесса может быть легко переформулировано на языке функционального интеграла. Пусть $A$ - любой функционал от случайного процесса $x(s)$. Определим среднее от $A$ формулой

$$
E(A)=(Z[F])^{-1} \int D x(s) A[x] e^{-S[x]}, \quad Z[F]=\int D x(s) e^{-S[x]},
$$

где $D x$ - винеровская мера, а $S$ - заданный функционал от $x$. Действие $S$ для любого гауссовского процесса имеет вид

$$
S[x]=\frac{1}{2} \int_{-\infty}^{\infty} d s \int_{-\infty}^{\infty} d s^{\prime} x(s) F\left(s, s^{\prime}\right) x\left(s^{\prime}\right) .
$$

Коррелятор координат равен $K(s, t)$, где $\int d s^{\prime} K\left(s, s^{\prime}\right) F\left(s^{\prime}, t\right)=\delta(s-t)$.

Действительно, для любой функции $g(s)$ и случайного гауссовского процесса $x(s)$ нетрудно проверить, что

$$
I[g] \stackrel{\text { def }}{=} E \exp \left(-\int_{-\infty}^{\infty} d s g(s) x(s)\right)=\exp \left(\frac{1}{2} \int_{-\infty}^{\infty} d s \int_{-\infty}^{\infty} d s^{\prime} g(s) K\left(s, s^{\prime}\right) g\left(s^{\prime}\right)\right) .
$$

С помощью этой формулы легко получить выражения (1) для средних. Например,

$$
E(x(t) x(s))=\left.\frac{\delta^{2}}{\delta g(s) \delta g(t)} I[g]\right|_{g=0}=K(t, s) .
$$

При явном нахождении преобразования Лапласа (2) ключевым моментом является тот факт, что среднее $Q_{\Lambda}(s)$ от функционалов вида

$$
\exp \left(-\frac{1}{2} \int_{-\infty}^{\infty} d s^{\prime} \int_{-\infty}^{\infty} d s^{\prime \prime} x\left(s^{\prime}\right) \Lambda\left(s^{\prime}, s^{\prime \prime} ; s\right) x\left(s^{\prime \prime}\right)\right), \quad \Lambda\left(s^{\prime}, s^{\prime \prime} ; s\right)=\Lambda\left(s^{\prime \prime}, s^{\prime} ; s\right),
$$

может быть вычислено с помощью формулы

$$
Q_{\Lambda}(s)=\exp \left(-\frac{1}{2} \int_{0}^{s} d s^{\prime} \int_{-\infty}^{\infty} d s^{\prime \prime} \int_{-\infty}^{\infty} d s^{\prime \prime \prime} R\left(s^{\prime \prime \prime}, s^{\prime \prime} ; s^{\prime}\right) \frac{\partial}{\partial s^{\prime}} \Lambda\left(s^{\prime \prime}, s^{\prime \prime \prime} ; s^{\prime}\right)\right),
$$

где функция $R$ удовлетворяет линейному интегральному уравнению

$$
R\left(s^{\prime}, s^{\prime \prime} ; s\right)=K\left(s^{\prime}, s^{\prime \prime}\right)-\int_{-\infty}^{\infty} d t^{\prime} \int_{-\infty}^{\infty} d t^{\prime \prime} K\left(s^{\prime}, t^{\prime}\right) \Lambda\left(t^{\prime}, t^{\prime \prime} ; s\right) R\left(t^{\prime \prime}, s^{\prime \prime} ; s\right) .
$$

Действительно, согласно (27) функцию $Q_{\Lambda}$ формально можно записать в виде

$$
Q_{\Lambda}(s)=\frac{Z[F+\Lambda]}{Z[F]}, \quad Z[F]=[\operatorname{det}(F)]^{-1 / 2} .
$$

Отсюда

$$
Q_{\Lambda}=e^{-\operatorname{Sp} \ln (1+\Lambda K) / 2} .
$$


Продифференцируем след от логарифма в (33) по $s$ в явном виде:

$$
\frac{\partial}{\partial s} \operatorname{Sp} \ln (1+\Lambda K)=\operatorname{Sp} R \frac{\partial}{\partial s} \Lambda, \quad R=K-K \Lambda K+K \Lambda K \Lambda K-\cdots .
$$

Интегрируя это выражение по $s$ и подставляя в формулу (33), имеем

$$
Q_{\Lambda}=\exp \left(-\frac{1}{2} \int_{0}^{s} d s^{\prime} \operatorname{Sp}\left(R \frac{\partial}{\partial s^{\prime}} \Lambda\right)\right), \quad R=K-K \Lambda R
$$

что совпадает с формулой (31).

Вычислим преобразование Лапласа (2). В этом случае

$$
\Lambda\left(s^{\prime}, s^{\prime \prime} ; s\right)=2 \lambda \delta\left(s^{\prime}-s^{\prime \prime}\right) \theta\left(s-s^{\prime}\right) .
$$

Уравнение (32) для $R$ сильно упрощается:

$$
R\left(s^{\prime}, s^{\prime \prime} ; s\right)=K\left(s^{\prime}, s^{\prime \prime}\right)-2 \lambda \int_{0}^{s} d t^{\prime} K\left(s^{\prime}, t^{\prime}\right) R\left(t^{\prime}, s^{\prime \prime} ; s\right) .
$$

Обозначая $\gamma(t, s)=2 \lambda R(t, s ; s)$, получим из формулы $(35)$, что

$$
\gamma(t, s)=2 \lambda K(t, s)-2 \lambda \int_{0}^{s} d x K(t, x) \gamma(x, s) .
$$

Тогда формулу (31) можно переписать в виде

$$
E e^{-\lambda \int_{0}^{s} d s^{\prime} x^{2}\left(s^{\prime}\right)}=e^{-\int_{0}^{s} d s^{\prime} \gamma\left(s^{\prime}, s^{\prime}\right) / 2} .
$$

Для того чтобы получить нелинейное уравнение (3), найдем производную от функции $R$ :

$$
\frac{\partial}{\partial k} R(t, s ; k)=-2 \lambda R(t, k ; k) R(k, s ; k) .
$$

По аналогии с диаграммной техникой уравнение (39) можно рассматривать как тождество Уорда для производной от “функции Грина” $R$. Уравнение (3) для $\gamma$ получается после интегрирования уравнения (39) при условии $t>s$ :

$$
\gamma(t, s)=2 \lambda K(t, s)-\int_{0}^{s} d k \gamma(t, k) \gamma(s, k) .
$$

Удивительно, что линейное интегральное уравнение (37) внешне совпадает с уравнением Гельфанда-Левитана-Марченко из метода обратной задачи рассеяния [7], однако ядра $K$, которые возникают при вычислении преобразования Лапласа (2), нетипичны для метода обратной задачи.

Покажем, как результаты раздела 1 получаются непосредственно из линейного уравнения (37). Пусть $K(t<s)=\Phi_{i}(t) \Psi_{i}(s)$. Тогда, записывая функцию Грина $\gamma(t<s)$ в виде $\gamma(t<s)=\phi_{j}(t) g_{j}(s)$, из уравнения (37) получим

$$
\phi_{j}(t) g_{j}(s)=\Phi_{i}(t) \Psi_{i}(s)-\Psi_{i}(t) \widetilde{R}_{i j}(t) g_{j}(s)+\Phi_{i}(t)\left[R_{i j}(t)-R_{i j}(s)\right] g_{j}(s),
$$


где

$$
R_{i j}(t)=2 \lambda \int_{0}^{t} d x \Psi_{i}(x) \phi_{j}(x), \quad \widetilde{R}_{i j}(t)=2 \lambda \int_{0}^{t} d x \Phi_{i}(x) \phi_{j}(x) .
$$

Определяя функции $\phi_{j}$ из линейных уравнений

$$
\phi_{j}(t)+2 \lambda \int_{0}^{t} d x\left[\Phi_{i}(x) \Psi_{i}(t)-\Phi_{i}(t) \Psi_{i}(x)\right] \phi_{j}(x)=\Phi_{j}(t),
$$

получим, что уравнение (41) сводится к соотношениям

$$
\Psi_{i}(s)=\left[\delta_{i j}+R_{i j}(s)\right] g_{j}(s),
$$

причем нетрудно проверить, что искомое выражение для $\gamma(s, s)$ имеет "солитонный” вид

$$
\gamma(s, s)=\frac{d}{d s} \ln \operatorname{det}\left[\delta_{i j}+R_{i j}(s)\right]
$$

Формула (38) дает простое выражение для искомого преобразования Лапласа (2):

$$
Q_{\lambda}(s)=\left(\operatorname{det}\left[\delta_{i j}+R_{i j}(s)\right]\right)^{-1 / 2} .
$$

Отметим, что “детерминантная" формула (45) совпадает с формулой (18), получающейся при решении нелинейного уравнения (3). Действительно, выразив при $2 \lambda=1$ матрицы $y_{1}, y_{2}$ через матрицы $\widetilde{R}$ и $R$ из (42), а вектор $h$ через вектор $\phi$ как

$$
y_{1}=\widetilde{R}, \quad y_{2}=1+R, \quad h=\phi,
$$

видим, что формулы (16) совпадают с (42), а уравнение (17) эквивалентно (43).

Благодарности. Авторы благодарны М.Л. Клепцыной за постановку задачи и многочисленные полезные обсуждения. Исследования были частично поддержаны грантом РФФИ № 08-01-00453-а, а также грантом НШ-3472.2008.2.

\section{Список литературы}

[1] M. L. Kleptsyna, A. Le Breton, Stoch. Stoch. Rep., 72:3-4 (2002), 229-250.

[2] M. L. Kleptsyna, P. E. Kloeden, V. V. Anh, Stochastic Anal. Appl., 16:5 (1998), 907-914.

[3] D. Revuz, M. Yor, Continuous Martingales and Brownian Motion, Grundlehren Math. Wiss., 293, Springer, Berlin, 1991.

[4] Р.Ш. Липцер, А.Н. Ширяев, Статистика случайных процессов, Наука, М., 1974.

[5] R. H. Cameron, W. T. Martin, Bull. Amer. Math. Soc., 51 (1945), 73-90.

[6] A. I. Yashin, J. Appl. Probab., 30:1 (1993), 247-251.

[7] В. Е. Захаров, С. В. Манаков, С. П. Новиков, Л. П. Питаевский, Теория солитонов: Метод обратной задачи, Наука, М., 1980. 